NASA/CR-1999-209345

ICASE Report No. 99-20

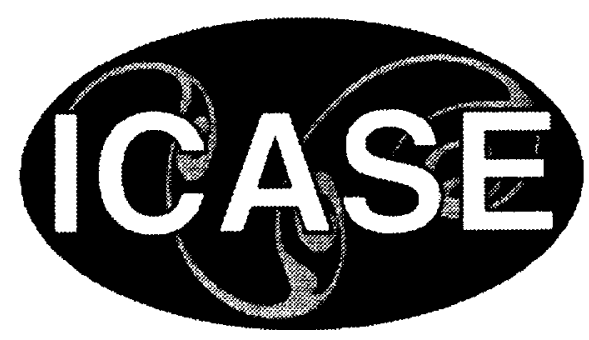

\title{
Factorizable Schemes for the Equations of Fluid Flow
}

David Sidilkover

ICASE, Hampton, Virginia

Institute for Computer Applications in Science and Engineering

NASA Langley Research Center

Hampton, VA

Operated by Universities Space Research Association

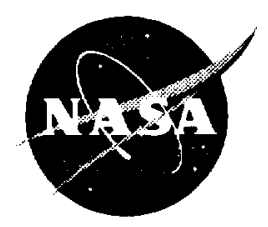

National Aeronautics and

Space Administration

Langley Research Center

Hampton, Virginia 23681-2199

Prepared for Langley Research Center under Contract NAS 1-97046

June 1999 
Available from the following:

NASA Center for AeroSpace Information (CASI) 7121 Standard Drive

Hanover, MD 21076-1320

(301) 621-0390
National Technical Information Service (NTIS)

5285 Port Royal Road

Springfield, VA 22161-2171

(703) $487-4650$ 


\title{
FACTORIZABLE SCHEMES FOR THE EQUATIONS OF FLUID FLOW
}

\author{
DAVID SIDILKOVER*
}

\begin{abstract}
We present an upwind high-resolution factorizable (UHF) discrete scheme for the compressible Euler equations that allows to distinguish between full-potential and advection factors at the discrete level. The scheme approximates equations in their general conservative form and is related to the family of genuinely multidimensional upwind schemes developed previously and demonstrated to have good shock-capturing capabilities. A unique property of this scheme is that in addition to the aforementioned features it is also factorizable, i.e. it allows to distinguish between full-potential and advection factors at the discrete level. The latter property facilitates the construction of optimally efficient multigrid solvers. This is done through a relaxation procedure that utilizes the factorizability property.
\end{abstract}

Key words. incompressible and compressible flow, factorizable schemes, genuinely multidimensional upwind schemes, optimal multigrid efficiency

Subject classification. Applied and Numerical Mathematics

1. Introduction. The standard numerical methods used for incompressible flow computations do not have much in common with the standard methods for compressible flow. The explanation of this fact is because difficulties encountered initially in each case were of a very different nature. We shall begin with briefly describing these difficulties and the evolution of the numerical methods for the two classes of problems.

1.1. Dimension-by-dimension methods (compressible flow). The main difficulty encountered when constructing numerical methods for compressible flow equations was the possible presence of discontinuities in the solutions. It took a prolonged effort of numerous researchers to devise what we call now the shock-capturing methodology. One of the basic ingredients of the shock-capturing schemes is the so-called (approximate) Riemann solvers (or their alternative - flux-splitting techniques) which are used to construct a first order accurate scheme. Another important ingredient is the so-called high-resolution mechanism, that allows one to combine higher order accuracy with shock-capturing capabilities, i.e., to circumvent Godunov's theorcm. It appears in the form of interpolation (or extrapolation) based on a certain smoothness monitor, that is usually implemented in the form of a flux-limiter. Many of the relevant issues could be studied on one-dimensional (unsteady) model problems.

The methods developed for one-dimensional problems were extended later to multidimensions in the most straightforward way - on a dimension-by-dimension basis.

One practical need was to compute steady flow, both external and internal, in multidimensions. Steady problems are generally solved through (pseudo-) time evolution. In other words, the problems are treated in a hyperbolic (with respect to time) sense. Multigrid methods became widely used as a mean to accelerate convergence to the steady-state. The key ingredient of a multigrid algorithm is the smoother, i.e., a relaxation procedure that efficiently reduces the high-frequency error content. The difficulty, however, is that the high-frequency error content may be nearly invisible in the residuals (poor measure of $h$-ellipticity) of highresolution schemes constructed on a dimension-by-dimension basis. This makes it inherently impossible to

*This work was supported by the National Acronautics and Space Administration under NASA Contract No. NAS1-97046 while the author was in residence at the Institute for Computer Applications in Science and Engineering (ICASE), Mail Stop 132C, NASA Langley Research Center, Hampton, VA 23681-2199, e-mail: sidilkovoicase.edu. 
construct an cfficient smoother.

Another major difficulty is that, in general, no distinction can be made between the different co-factors at the discrete levcl, i.c., the standard high-resolution schemes are not factorizable. This deficiency of the standard discretizations also contributes to the poor performance of standard multigrid solvers. It also leads to the loss of accuracy and deteriorating computational efficiency in the case of low speed compressible flow.

Discrete schemes that are only partially based on the dimension-by-dimension approach were presented in [6], [7], [9] and [13]. In these schemes, some of the second order corrections appear in the form of terms approximating mixed derivatives. The remaining corrections are based on dimension-by-dimension highresolution mechanism. Schemes of this type when tuned for steady-state computations would have a better $h$-ellipticity property than the standard ones. This direction, however, has not been explored.

1.2. Projection-type methods (incompressible flow). Incompressible flow in one dimension is trivial. Therefore, numerical analysts had to address the multidimensional problems right away. Incompressible flow problems (both steady and unsteady) contain an elliptic part, which rules out the use of an explicit scheme.

One of the earlier approaches was to substitute the continuity equations by the pressure Poisson equation, derived using the momentum and the continuity equations (see a summary in [10]). By using such a representation, decoupling of the elliptic factor (in the form of the pressure Poisson equation) from the rest of the system can be achieved. This formulation has been used in conjunction with multigrid as well. In [20], it was demonstrated that by deriving and treating the boundary conditions for the pressure properly one can obtain the ideal multigrid efficiency both for inviscid and viscous cases. Some different versions of the algorithm are discussed in [15] and in [14], where some steps in deriving the pressure Poisson equation are made at the discrete level. Two variants of the algorithm are being pursued: one for structured body-fitted grids, another for unstructured grids (see [14]). Ideal multigrid efficiency was demonstrated for both on several test cases. A possible extension of this approach to the compressible subsonic case is discussed in $[15]$.

Several methods exist based on discretizing the equations of incompressible flow in their usual (primitive) form. It is interesting to note that one of the earlier methods (MAC) (see [8]) relies on a staggered grid discretization and uses a pressure Poisson equation derived at the discrete level to update the pressure.

A known property of a vector field is that it can be represented as a sum of its irrotational and solenoidal components. The attractive feature of the staggered grid discretization is that this property can be imitated on the discrete level (second-order accurate approximation to the Cauchy-Riemann equations) without producing an odd-even instability. It is problematic, though, to achieve the same on non-staggered grids.

When solving the equations of incompressible flow in their primitive form, the important part of the process is to satisfy the continuity equation, i.e., to project the velocity field onto the divergence-free manifold or, in other words, to discard the irrotational component of the velocity field. Such a process is the main part of the projection method [5], whose original version was based on non-staggered grids. It was reformulated later using staggered grids. A convenient way to perform the projection step is to introduce an auxiliary variable potential and to solve the resulting Poisson equation. This was first done in [1].

A commonly used algorithm for solving the incompressible flow equations is the SIMPLE algorithm by Patankar \& Spalding [12]. It is interesting to note that this algorithm was extended to the compressible (subsonic) case (see [11],[2]).

Applying multigrid methods for solving the fluid flow problems is one of the main subjects of the landmark work [3]. Although a few various possibilities are mentioned, the approach, systematically studied 
and advocated, is based on the staggered-grid discretization and an auxiliary potential variable. It was shown that the proper treatment (Distributive Gauss-Seidcl relaxation - shortly DGS) results in the decoupling of different co-factors of the system. It was demonstrated later in [4] that an optimal MG efficiency can be obtained for the incompressible Euler equations. Some ways of generalizing these ideas to the compressible flow were sketched out in $[3]$ as well.

The Vorticity-Potential (or vorticity and streamfunction in two dimensions) formulation of the flow equations seems very attractive, since the different co-factors of the equations decouple. The well known difficulty associated with this formulation, though, is the derivation and treatment of the boundary conditions for vorticity. Another difficulty associated with this approach is that the components of the vorticity vector in three dimensions need to satisfy a certain compatibility condition. Also, it is highly problematic to obtain a numerical scheme with shock-capturing capabilities using the Vorticity-Potential formulation.

The ideal multigrid efficiency for the compressible Euler equations in the subsonic case was demonstrated first in [23] using the canonical variables formulation of the equations [22]. However, this formulation cannot be generalized to viscous and unsteady cases.

1.3. About this paper. In order to achieve optimal multigrid efficiency, we have to use projectiontype methods to solve the discrete equations, i.e., methods that distinguish between the different co-factors of the equations.

The attempts $([2],[23])$ to apply projection-type methods to solve the compressible flow equations were limited to the subsonic case, since the discretizations used have no shock-capturing capabilities. On the other hand the projection-type methods cannot be applied in conjunction with the standard upwind dimensionby-dimension discretizations, since the latter are not factorizable (see [15]), i.e., no distinction between the different factors can be made at the discrete level.

There is a need for a factorizable discretization scheme with good shock-capturing capabilities. The scheme should rely on a high resolution mechanism and it should be sensitive to the high-frequency error content ( $h$-elliptic) at the same time. The flow equations should also be approximated in their usual conservative form, since using special formulations leads to the loss of generality. The separation between the treatment of co-factors should be achieved through the relaxation procedure, which may rely on some special auxiliary variables and/or special forms of the equations. It may look as if too many (seemingly contradictory) requirements need to be satisficd by a single discretization. The purpose of this paper is to describe a construction of such a discrete scheme.

A search for a genuinely multidimensional upwind scheme was motivated initially by the necessity to devise a discretization that has the high-resolution and $h$-ellipticity property at the same time. Such a scheme for scalar advection problems was proposed in [16],[21]. This approach was extended to the Euler equations in $[18,17,19]$ in the context of unstructured triangular grids. It was demonstrated that a simple pointwise Gauss-Seidel relaxation is stable when applicd directly to the high-resolution discrete equation (contrary to the case of the standard schemes) and has good smoothing properties. The latter is a manifestation of the $h$-ellipticity property of the discretization.

It was pointed out in [15] that the genuinely multidimensional approach towards discretizing the compressible flow equations leads to schemes that are, in addition to other desirable properties, factorizable. In this paper we describe such a scheme, i.e. a scheme which is Upwind, High-resolution and Factorizable (UHF). Also, we develop a relaxation procedure that utilizes the factorizability property of the scheme.

2. Preliminaries. In this section we briefly review the genuinely two-dimensional advection scheme, introduce some basic properties of the Euler cquations and discuss some standard discretization schemes for 


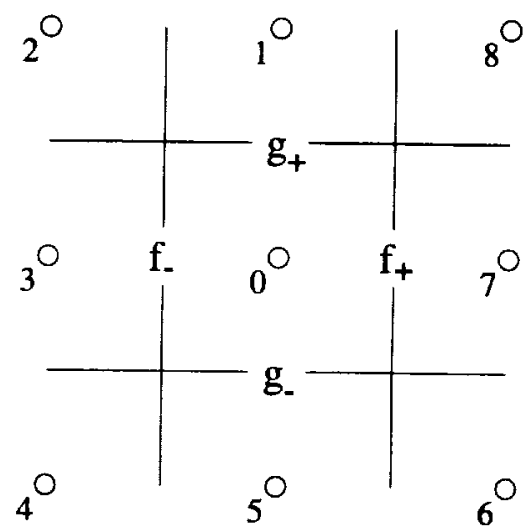

FIG. 1. Computational grid segment and a control volume.

the fluid flow equations.

2.1. Scalar advection schemes. Consider a steady-state conservation law in two dimensions

$$
-\varepsilon \Delta u+f(u)_{x}+g(u)_{y}=0
$$

where $\varepsilon>0$ is an infinitesimally small number. A general conservative discretization of (2.1) is given by

$$
h\left[\left(f_{+}-f_{-}\right)+\left(g_{+}-g_{-}\right)\right]=0
$$

where $f_{ \pm}, g_{ \pm}$are the numerical fluxes. A particular discretization scheme for (2.1) can be given by defining these numerical fluxes.

A numerical scheme can be written in the form

$$
u_{0}=\sum_{i} C_{i} u_{i}
$$

DEFINITION 2.1. The scheme is said to be of the positive type if $C_{i} \geq 0$.

We shall illustrate the construction of some discretization scheme on the linear constant coefficient version of $(2.1)$

$$
-\varepsilon \Delta u+a u_{x}+b u_{y}=0 .
$$

A first order upwind scheme can be given by the following numerical fluxes

$$
\begin{aligned}
& f_{-}^{u}=\frac{1}{2} a\left(u_{0}+u_{3}\right)-\frac{1}{2}|a|\left(u_{0}-u_{3}\right) \\
& g_{-}^{u}=\frac{1}{2} b\left(u_{0}+u_{5}\right)-\frac{1}{2}|b|\left(u_{0}-u_{5}\right) .
\end{aligned}
$$

Assume for simplicity that

$$
b \geq a \geq 0 .
$$

A second order scheme with a genuinely multidimensional flavor is given by

$$
\begin{aligned}
& f_{-}=f_{-}^{u}-\frac{1}{2} b\left(u_{3}-u_{4}\right) \\
& g_{-}=g_{-}^{u}-\frac{1}{2} a\left(u_{5}-u_{4}\right) .
\end{aligned}
$$

This scheme, however, is not of the positive type. 
In order to combine the positivity property with the second order accuracy we need to incorporate the second order correction in a nonlinear fashion. A "straightforward" way of doing this is to modify the fluxes in the following way

$$
\begin{aligned}
& f_{-}=f_{-}^{u}-\frac{1}{2} b\left(u_{3}-u_{4}\right) \psi\left(Q_{-}\right) \\
& g_{-}=g_{-}^{u}-\frac{1}{2} a\left(u_{5}-u_{4}\right) \psi\left(S_{-}\right),
\end{aligned}
$$

which gives a positive second order scheme if the limiter function $\psi$ satisfies the inequality

$$
\begin{gathered}
0 \leq \psi(Q) \leq 1 ; \quad 0 \leq \frac{\psi(Q)}{Q} \leq 1, \\
\psi(1)=1
\end{gathered}
$$

and the arguments of the limiter function are defined by

$$
\begin{gathered}
Q_{-}=-\frac{a\left(u_{0}-u_{3}\right)}{b\left(u_{3}-u_{4}\right)} \\
S_{-}=-\frac{b\left(u_{0}-u_{5}\right)}{a\left(u_{5}-u_{4}\right)} .
\end{gathered}
$$

Using the following identities

$$
\begin{aligned}
& b\left(u_{3}-u_{4}\right) \psi\left(Q_{-}\right) \equiv-a\left(u_{0}-u_{3}\right) \psi\left(Q_{-}\right) / Q_{-} \\
& a\left(u_{5}-u_{4}\right) \psi\left(S_{-}\right) \equiv-b\left(u_{0}-u_{5}\right) \psi\left(S_{-}\right) / R_{-}
\end{aligned}
$$

it is easy to see that this scheme is no better than the standard high-resolution schemes, since under certain circumstances it becomes identical to a central scheme (no $h$-ellipticity property). The fact that it is positive for a non-compressive limiter only (i.c., no artificial compression can be added) is a disadvantage as well.

Note, that a part of the second order correction can be added with no limiter while still resulting in a positive (first order) scheme - the so-called $N$-scheme (see $[16,21]$ )

$$
\begin{aligned}
& f_{-}^{N}=f_{-}^{u}-\frac{1}{2} a\left(u_{3}-u_{4}\right) \\
& g_{-}^{N}=g_{-}^{u}-\frac{1}{2} a\left(u_{5}-u_{4}\right)
\end{aligned}
$$

A second order high-resolution scheme can be obtained by adding the remaining part of the correction in a nonlinear fashion

$$
\begin{aligned}
& f_{-}=f_{-}^{N}-\frac{1}{2}(b-a)\left(u_{3}-u_{4}\right) \psi\left(R_{-}\right) \\
& g_{-}=g_{-}^{N}
\end{aligned}
$$

where

$$
R_{-}=-\frac{a\left(u_{5}-u_{4}\right)}{b\left(u_{3}-u_{4}\right)}
$$

It is easy to see $([21])$ that $(2.12)$ defines a positive scheme if

$$
0 \leq \psi(R) \leq 2 ; \quad 0 \leq \frac{\psi(R)}{R} \leq 2
$$


2.2. Euler equations. The quasilinear form of the steady Euler equations in two dimensions can be written in the following way

$$
L \mathbf{u}=0
$$

where $\mathbf{u}=(s, u, v, p)^{T}$ is the vector of unknown functions (namely, entropy, horizontal velocity component, vertical velocity component and pressure, respectively),

$$
L=\left(\begin{array}{cccc}
q & 0 & 0 & 0 \\
0 & \rho q & 0 & \partial_{x} \\
0 & 0 & \rho q & \partial_{x} \\
0 & \rho c^{2} \partial_{x} & \rho c^{2} \partial_{y} & q
\end{array}\right)
$$

$\rho$ stands for density, $c$ for the speed of sound and $q$ denotes the advection operator (with velocity $(u, v)$ ). We shall consider the case of subsonic flow $\left(u^{2}+v^{2} \leq c^{2}\right)$ throughout the paper. It is sufficient for the purpose of the analysis in this paper to consider the linear constant coefficient case.

Determining the type of a system of partial differential equations can be done formally by computing the determinant of matrix $L$ and examining its principal part

$$
\operatorname{det} L=(\rho q)^{2} \cdot\left(q^{2}-c^{2} \Delta\right)
$$

The first multiplier in (2.16) is the advection operator (times the density) in the power equal to the dimension of the problem. The second multiplier is the full-potential operator, which, in subsonic case, is of elliptic type. Thus, the steady Euler system of equations is of the mixed hyperbolic-elliptic type.

2.2.1. One-dimensional case. Consider a first order upwind scheme for the one-dimensional Euler equations. Without loss of generality we consider the primitive variable formulation

$$
L \mathbf{u}=\mathbf{0}
$$

where $\mathbf{u}=(s, u, p)^{T}$ and

$$
L=\left(\begin{array}{ccc}
u \partial_{x} & 0 & 0 \\
0 & \rho u \partial_{x} & \partial_{x} \\
0 & \rho c^{2} \partial_{x} & u \partial_{x}
\end{array}\right)
$$

A first order upwind scheme approximating (2.17) is given by

$$
L^{h} \mathbf{u}^{h}=0
$$

where the discrete variables

$$
\mathbf{u}^{h}=\left(s^{h}, u^{h}, p^{h}\right)^{T}
$$

and

$$
L^{h}=\left(\begin{array}{ccc}
-\frac{h}{2}|u| \partial_{x x}^{h}+Q^{2 h} & 0 & 0 \\
0 & \rho\left(-\frac{h}{2} c \partial_{x x}^{h}+Q^{2 h}\right) & \partial_{x}^{2 h}-\frac{h}{2} \frac{u}{c} \partial_{x x}^{h} \\
0 & \rho c^{2}\left(\partial_{x}^{2 h}-\frac{h}{2} \frac{u}{c} \partial_{x x}^{h}\right) & -\frac{h}{2} c \partial_{x x}+Q^{2 h}
\end{array}\right)
$$

where $h$ is a mesh size, $\partial_{x x}^{h}$ is a central approximation of the second derivative, $\partial_{x}^{2 h}$ is a central approximation of the first derivative and $Q^{2 h}=u \partial_{x}^{2 h}$ is the advection operator. 
Factorization. The determinant of $L^{h}$ :

$$
\operatorname{det}\left(L^{h}\right)=\rho\left(-\frac{h}{2}|u| \partial_{x x}^{h}+Q^{2 h}\right)\left[\left(c^{2}-u^{2}\right) \partial_{x x}^{h}\right]
$$

The first factor is the upwind scheme approximating the advection operator corresponding to the entropy equation. The Full-Potential factor is approximated by a "short" central difference. The issue of factorization appears to be trivial in this case, since the momentum and the pressure equations correspond solely to the elliptic factor.

2.2.2. Two-dimensional case. Since the equation for entropy decouples in the form of the advection equation, whose numerical treatment is straightforward, we can consider without loss of generality the case of isentropic flow. We can also assume for simplicity that $\rho=1$. Two-dimensional steady isentropic linearized Euler equations are now given by the following

$$
L \mathbf{u}=\mathbf{0}
$$

where $\mathbf{u}=(u, v, p)^{T}$ and

$$
L=\left(\begin{array}{ccc}
q & 0 & \partial_{x} \\
0 & q & \partial_{y} \\
c^{2} \partial_{x} & c^{2} \partial_{y} & q
\end{array}\right)
$$

The FDA (First Differential Approximation or Modified equations) corresponding to the first order upwind scheme, constructed on the dimension-by-dimension basis, is given by

$$
L_{1}^{F D A}=\left(\begin{array}{ccc}
\left(\mathbf{q}-\frac{h}{2}(c-|u|) \partial_{x x}\right) & 0 & \partial_{x}-\frac{h}{2} \frac{u}{c} \partial_{x x} \\
0 & \left(\mathbf{q}-\frac{h}{2}(c-|v|) \partial_{y y}\right) & \partial_{y}-\frac{h}{2} \frac{v}{c} \partial_{y y} \\
c^{2}\left(\partial_{x}-\frac{h}{2} \frac{u}{c} \partial_{x x}\right) & c^{2}\left(\partial_{y}-\frac{h}{2} \frac{v}{c} \partial_{y y}\right) & Q-\frac{h}{2} c \Delta
\end{array}\right)
$$

where $\mathbf{q}$ is an FDA of the first order upwind advection scheme. It is easy to verify that $L_{1}^{F D A}$ does not preserve the factorizability property of $L$, i.e., in the $\operatorname{det}\left(L_{1}^{F D A}\right)$ we cannot distinguish between the FDAs corresponding to advection and full-potential factors. The same applies, of course, to the corresponding discrete scheme.

The dimension-by-dimension scheme (whose FDA is given by (2.25)) can be upgraded to higher order accuracy by introducing the standard nonlinear high-resolution correction. However, by doing so, we obtain a scheme that is not only not factorizable but also can be insensitive to some high-frequency error components (has a poor measure of $h$-ellipticity). All this makes it practically impossible to construct an efficient solver for the resulting discrete equations.

3. Factorizable scheme. In order to combine the high-resolution and $h$-ellipticity properties the extension of the ideas leading to the genuinely multidimensional advection scheme need to be generalized to systems of equations. Such a generalization on triangular grids was proposed in $[18,17,19]$. As well as in the scalar case, the second-order corrections are given by the terms approximating mixed derivatives. These corrections may rely on two-dimensional limiters. It was demonstrated that the Gauss-Seidel relaxation is stable when applied directly to the high-resolution discrete equations (unlike in the case of standard dimcnsion-by-dimension schemes). This indicated that these new schemes have stability properties superior to the standard ones.

It was pointed out in [17] that some of these corrections can be added without limiters, in a linear fashion. Indeed, similar to the scalar case (see [21] and also §2.1), in order to obtain a scheme with better 
linear stability properties, it is desirable to include as much of the second order correction as possible without limiters. This is especially important in the context of structured grids ("9-point box" stencil); otherwise one can obtain a scheme that is no better than the standard ones (see $\$ 2.1$ ).

However, unlike the scalar case, it is unclear what is a suitable criterion for determining which parts of the corrections do not have to rely on the limiters. The difficulty here is that the notions of maximum principle and positivity do not extend to systems of equations in a suitable way.

We recall here the observation that was made in [15]. Consider the FDA corresponding to the second order genuinely multidimensional upwind scheme with all the second order corrections added in a linear fashion

$$
L_{2}^{F D A}=\left(\begin{array}{rrr}
\mathbf{q}-\frac{h}{2}(c-|u|) \partial_{x x} & -\frac{h}{2}(c-|v|) \partial_{x y} & \partial_{x}-\frac{h}{2} \frac{1}{c} Q_{x} \\
-\frac{h}{2}(c-|u|) \partial_{x y} & \mathbf{q}-\frac{h}{2}(c-|v|) \partial_{y y} & \partial_{y}-\frac{h}{2} \frac{1}{c} Q_{y} \\
c^{2} \partial_{x}-\frac{h}{2} c Q_{x} & c^{2} \partial_{y}-\frac{h}{2} c Q_{y} & Q-\frac{h}{2} c \Delta
\end{array}\right)
$$

It can be verified that (3.1) is factorizable.

The implication of this is that the multidimensional corrections not only lead to a second-order scheme, but some of them are also responsible for the factorizability. Since it is a desirable property, the latter corrections should be included without limiters.

There exist many discretizations that correspond to (3.1). Note that not all of them are factorizable. We would now like to construct an actual discrete scheme which is factorizable as well.

The technical difficulty here is that the following differential equalities

$$
\begin{aligned}
& \partial_{x x} \partial_{y y}=\partial_{x y} \partial_{x y} \\
& \partial_{x x} \partial_{y}=\partial_{x y} \partial_{x} \\
& \partial_{y y} \partial_{x}=\partial_{x y} \partial_{y}
\end{aligned}
$$

need to have discrete analogs. Approximating the derivatives $\partial_{x}, \partial_{x x}, \partial_{y}, \partial_{y y}$ by standard central finite differences and discretizing the mixed derivatives in some reasonable way, we can see that the discrete analog of (3.2) does not hold.

Let us introduce some non-standard finite differences

$$
\begin{gathered}
\bar{\partial}_{x}=\frac{1}{8} \frac{1}{h}\left(\begin{array}{rrr}
-1 & 0 & 1 \\
-2 & 0 & 2 \\
-1 & 0 & 1
\end{array}\right) ; \quad \bar{\partial}_{y}=\frac{1}{8} \frac{1}{h}\left(\begin{array}{rrr}
1 & 2 & 1 \\
0 & 0 & 0 \\
-1 & -2 & -1
\end{array}\right) \\
\bar{\partial}_{x x}=\frac{1}{4} \frac{1}{h^{2}}\left(\begin{array}{rrr}
1 & -2 & 1 \\
2 & -4 & 2 \\
1 & -2 & 1
\end{array}\right) ; \quad \bar{\partial}_{y y}=\frac{1}{4} \frac{1}{h^{2}}\left(\begin{array}{rrr}
1 & 2 & 1 \\
-2 & -4 & -2 \\
1 & 2 & 1
\end{array}\right) \\
\bar{\partial}_{x y}=\frac{1}{4} \frac{1}{h^{2}}\left(\begin{array}{rrr}
-1 & 0 & 1 \\
0 & 0 & 0 \\
1 & 0 & -1
\end{array}\right) .
\end{gathered}
$$

Let us denote also

$$
\bar{\Delta}=\frac{1}{4} \frac{1}{h^{2}}\left(\begin{array}{rrr}
1 & 2 & 1 \\
2 & -12 & 2 \\
1 & 2 & 1
\end{array}\right)
$$


and

$$
\begin{aligned}
& \bar{Q}_{x}^{h}=u \bar{\partial}_{x x}+v \bar{\partial}_{x y} \\
& \bar{Q}_{x}^{h}=u \bar{\partial}_{x y}+v \bar{\partial}_{y y} .
\end{aligned}
$$

It is easy to see that the discrete analog of (3.2) holds if the derivatives are approximated by the finite differences as defined by (3.3), (3.4) and (3.5).

Consider now the following discrete scheme that corresponds to the FDA given by (3.1)

$$
L^{h}=\left(\begin{array}{rrr}
\mathbf{q}^{h}-\frac{h}{2}(c-|u|) \bar{\partial}_{x x}^{h} & -\frac{h}{2}(c-|v|) \bar{\partial}_{x y}^{h} & \bar{\partial}_{x}^{h}-\frac{h}{2} \frac{1}{c} \bar{Q}_{x}^{h} \\
-\frac{h}{2}(c-|u|) \bar{\partial}_{x y}^{h} & \mathbf{q}^{h}-\frac{h}{2}(c-|v|) \bar{\partial}_{y y}^{h} & \bar{\partial}_{y}^{h}-\frac{h}{2} \frac{1}{c} \bar{Q}_{y}^{h} \\
c^{2} \partial_{x}^{h}-\frac{h}{2} c Q_{x} & c^{2} \partial_{y}^{h}-\frac{h}{2} c Q_{y} & Q^{h}-\frac{h}{2} c \bar{\Delta}^{h}
\end{array}\right)
$$

It is factorizable and its determinant is given by

$$
\begin{aligned}
\operatorname{det}\left(L^{h}\right)=\mathbf{q}^{h}\left[( Q ^ { h } - \frac { h } { 2 } c \overline { \Delta } ^ { h } ) \left(\mathbf{q}^{h}-\right.\right. & \left.\frac{h}{2}(c-|u|) \bar{\partial}_{x x}^{h}-\frac{h}{2}(c-|v|) \bar{\partial}_{y y}^{h}\right) \\
& -\left(\bar{\partial}_{x}^{h}-\frac{h}{2} \frac{1}{c} \bar{Q}_{x}^{h}\right)\left(c^{2} \partial_{x}^{h}-\frac{h}{2} c Q_{x}\right) \\
& \left.-\left(\bar{\partial}_{y}^{h}-\frac{h}{2} \frac{1}{c} \bar{Q}_{y}^{h}\right)\left(c^{2} \partial_{y}^{h}-\frac{h}{2} c Q_{y}\right)\right]
\end{aligned}
$$

Note that the discrete approximation of the elliptic factor becomes the so-called "skewed" discrete Laplacian (not an $h$-elliptic operator) when the flow speed (Mach number) goes to zero. This difficulty can be dealt with in various ways. The simplest option is to slightly modify the scheme so that the problem mentioned above disappears.

Denote the total velocity

$$
U=\left|\sqrt{u^{2}+v^{2}}\right|
$$

the Mach number

$$
M=\frac{U}{c}
$$

and the "augmented" Mach number

$$
\bar{M}= \begin{cases}M, & \text { if } M>C h \\ C h & \text { otherwise }\end{cases}
$$

where $C>0$ is a constant.

We can "rescale" the artificial dissipation terms, obtaining the following discrete scheme, which we shall call UHF (Upwind, High-resolution, Factorizable)

$$
L=\left(\begin{array}{rrr}
\overline{\mathbf{q}}^{h}-\frac{h}{2}(U-|u|) \bar{\partial}_{x x}^{h} & -\frac{h}{2}(U-|v|) \bar{\partial}_{x y}^{h} & \bar{\partial}_{x}^{h}-\frac{h}{2} \frac{M}{c} \bar{Q}_{x}^{h} \\
-\frac{h}{2}(U-|u|) \bar{\partial}_{x y}^{h} & \overline{\mathbf{q}}^{h}-\frac{h}{2}(U-|v|) \bar{\partial}_{y y}^{h} & \bar{\partial}_{y}^{h}-\frac{h}{2} \frac{M}{c} \bar{Q}_{y}^{h} \\
c^{2}\left(\partial_{x}^{h}-\frac{h}{2} \frac{1}{c M} Q_{x}\right) & c^{2}\left(\partial_{y}^{h}-\frac{h}{2} \frac{1}{c M} Q_{y}\right) & Q^{h}-\frac{h}{2} \frac{c}{M} \bar{\Delta}^{h}
\end{array}\right)
$$

where

$$
\overline{\mathbf{q}}^{h}=\mathbf{q}^{h}-\frac{1}{2} h^{2} c C \Delta^{h}
$$


is the discrete advection operator augmented by a second-order small dissipative term and $\Delta^{h}$ is the usual "5-point-star" discrete Laplacian. This "regularization," obviously, prevents the advection scheme from degenerating at a stagnation point. It plays an additional important role, though, which will be explained later in this section.

Before discussing some properties of the UHF scheme and computing its determinant, we shall introduce the simplified notation

$$
\begin{aligned}
& \boldsymbol{\partial}_{x}^{h}=\partial_{x}^{h}-\frac{h}{2} \frac{1}{c M} Q_{x}^{h} \\
& \boldsymbol{\theta}_{y}^{h}=\partial_{y}^{h}-\frac{h}{2} \frac{1}{c \bar{M}} Q_{y}^{h} \\
& \overline{\boldsymbol{\theta}}_{x}^{h}=\bar{\partial}_{x}^{h}-\frac{h}{2} \frac{M}{c} \bar{Q}_{x}^{h} \\
& \overline{\boldsymbol{\partial}}_{y}^{h}=\bar{\partial}_{y}^{h}-\frac{h}{2} \frac{M}{c} \bar{Q}_{y}^{h}
\end{aligned}
$$

and

$$
\begin{gathered}
\tilde{\mathbf{q}}^{h}=Q^{h}-\frac{h}{2} \frac{c}{\bar{M}} \bar{\Delta}^{h}, \\
\hat{\mathbf{q}}^{h}=\overline{\mathbf{q}}^{h}-\frac{h}{2}\left((U-|u|) \bar{\partial}_{x x}^{h}+(U-|v|) \bar{\partial}_{y y}^{h}\right) .
\end{gathered}
$$

Then the UHF scheme (3.10) can be written as

$$
L=\left(\begin{array}{rrr}
\overline{\mathbf{q}}^{h}-\frac{h}{2}(U-|u|) \bar{\partial}_{x x}^{h} & -\frac{h}{2}(U-|v|) \bar{\partial}_{x y}^{h} & \overline{\boldsymbol{\theta}}_{x}^{h} \\
-\frac{h}{2}(U-|u|) \bar{\partial}_{x y}^{h} & \overline{\mathbf{q}}^{h}-\frac{h}{2}(U-|v|) \bar{\partial}_{y y}^{h} & \overline{\boldsymbol{\theta}}_{y}^{h} \\
c^{2} \boldsymbol{\partial}_{x}^{h} & c^{2} \boldsymbol{\partial}_{y}^{h} & \tilde{\mathbf{q}}^{h}
\end{array}\right)
$$

and its determinant

$$
\operatorname{det}\left(L^{h}\right)=\overline{\mathbf{q}}^{h} \cdot\left[c^{2}\left(\boldsymbol{\theta}_{x}^{h} \overline{\boldsymbol{\theta}}_{x}^{h}+\boldsymbol{\partial}_{y}^{h} \overline{\boldsymbol{\theta}}_{y}^{h}\right)-\tilde{\mathbf{q}}^{h} \cdot \hat{\mathbf{q}}^{h}\right]
$$

Let us take a closer look at what happens at a stagnation point $(U=u=v=0)$

$$
\begin{aligned}
& \overline{\mathbf{q}}^{h}=-\frac{1}{2} h^{2} c C \Delta^{h} \\
& \hat{\mathbf{q}}^{h}=-\frac{1}{2} h^{2} c C \Delta^{h} \\
& \tilde{\mathbf{q}}^{h}=-\frac{c}{2 C} \bar{\Delta}^{h} .
\end{aligned}
$$

It becomes plain that

$$
\tilde{\mathbf{q}}^{h} \cdot \hat{\mathbf{q}}^{h}=\frac{1}{4} h^{2} c^{2} \bar{\Delta}^{h} \Delta^{h}
$$

which is an $h$-elliptic operator. We can conclude now that for the UHF scheme (3.16) (or (3.10)) the approximation of the Full-Potential factor remains $h$-elliptic in the case of vanishing velocity. Moreover, it can be verified that the Full-Potential factor is approximated in this case by a "regular" five-point star discretc Laplacian $\Delta^{h}$. 
4. The relaxation procedure. Having constructed a factorizable discretization scheme for the Euler equations, an important question becomes how to use this property in order to obtain an efficient algorithm for solving the discrete equations.

Introduce the auxiliary variables, namely, the discrete stream-function and potential by the following

$$
\left(\begin{array}{c}
u^{h} \\
v^{h} \\
p^{h}
\end{array}\right)=\mathcal{M}^{h} \cdot\left(\begin{array}{c}
\Psi^{h} \\
\Phi^{h}
\end{array}\right)
$$

where

$$
\mathcal{M}^{h}=\left(\begin{array}{rr}
\boldsymbol{\partial}_{y} & \overline{\boldsymbol{\theta}}_{x}^{h} \\
-\boldsymbol{\partial}_{x}^{h} & \overline{\boldsymbol{\theta}}_{y}^{h} \\
0 & \hat{\mathbf{q}}^{h}
\end{array}\right)
$$

We can also define the discrete vorticity

$$
\omega^{h}=\operatorname{curl}^{h} \cdot\left(u^{h}, v^{h}\right)^{T}=\left(\overline{\boldsymbol{D}}_{y}^{h},-\overline{\boldsymbol{D}}_{x}^{h}\right) \cdot\left(u^{h}, v^{h}\right)^{T}
$$

or

$$
\omega^{h}=\left(\bar{\partial}_{x}^{h} \partial_{x}^{h}+\bar{\partial}_{y}^{h} \partial_{y}^{h}\right) \Psi^{h}
$$

Introduce also the following matrix operator

$$
\mathcal{P}^{h}=\left(\begin{array}{ccc}
\overline{\boldsymbol{\partial}}_{y}^{h} & -\ddot{\boldsymbol{\theta}}_{x}^{h} & 0 \\
0 & 0 & 1
\end{array}\right)
$$

It is easy to see that applying the discrete $\overrightarrow{c u r l}^{h}$ operator to the momentum equations (pre-multiplying $L^{h}$ by $\mathcal{P}^{h}$ ) and performing the substitution of variables according to (4.1) (post-multiplying $L^{h}$ by $\mathcal{M}^{h}$ ), we obtain

$$
\mathcal{P}^{h} \cdot L^{h} \cdot \mathcal{M}^{h}=\left(\begin{array}{cc}
\overline{\mathbf{q}}^{h} \cdot\left(\boldsymbol{\partial}_{x}^{h} \overline{\boldsymbol{\partial}}_{x}^{h}+\boldsymbol{\partial}_{y}^{h} \overline{\boldsymbol{\partial}}_{y}^{h}\right) & 0 \\
0 & c^{2}\left(\boldsymbol{\partial}_{x}^{h} \overline{\boldsymbol{\partial}}_{x}^{h}+\boldsymbol{\partial}_{y}^{h} \overline{\boldsymbol{\partial}}_{y}^{h}\right)-\tilde{\mathbf{q}}^{h} \cdot \hat{\mathbf{q}}^{h}
\end{array}\right) .
$$

In other words we end up with "solving" the system

$$
\left(\begin{array}{cc}
\overline{\mathbf{q}}^{h} \cdot\left(\boldsymbol{\partial}_{x}^{h} \overline{\boldsymbol{\partial}}_{x}^{h}+\boldsymbol{\partial}_{y}^{h} \overline{\boldsymbol{\partial}}_{y}^{h}\right) & 0 \\
0 & c^{2}\left(\boldsymbol{\partial}_{x}^{h} \overline{\boldsymbol{\partial}}_{x}^{h}+\boldsymbol{\partial}_{y}^{h} \overline{\boldsymbol{\partial}}_{y}^{h}\right)-\tilde{\mathbf{q}}^{h} \cdot \hat{\mathbf{q}}^{h}
\end{array}\right) \cdot\left(\begin{array}{c}
\Psi^{h} \\
\Phi^{h}
\end{array}\right)=\mathbf{0}
$$

or

$$
\left(\begin{array}{cc}
\overline{\mathbf{q}}^{h} & 0 \\
0 & c^{2}\left(\boldsymbol{\partial}_{x}^{h} \overline{\boldsymbol{\partial}}_{x}^{h}+\boldsymbol{\partial}_{y}^{h} \overline{\boldsymbol{\partial}}_{y}^{h}\right)-\tilde{\mathbf{q}}^{h} \cdot \hat{\mathbf{q}}^{h}
\end{array}\right) \cdot\left(\begin{array}{c}
\omega^{h} \\
\Phi^{h}
\end{array}\right)=0 .
$$

The Distributive relaxation procedure at a point amounts to computing updates to the discrete potential and vorticity (or streamfunction) according to (4.8) and to translating them into corresponding updates of the velocity components and the pressure at the point of interest and its neighbors according to matrix (4.2) and (4.1).

Note that the operator $\left(\boldsymbol{\partial}_{x}^{h} \overline{\boldsymbol{\partial}}_{x}^{h}+\boldsymbol{\partial}_{y}^{h} \overline{\boldsymbol{\partial}}_{y}^{h}\right)$ is not $h$-elliptic. Therefore, the relaxation procedure described above will not smooth certain high-frequency error components. This lack of $h$-ellipticity seems unavoidable for obtaining the desired factorization. However, a simple remedy exits for this trouble. It is to augment each sweep of the Distributive relaxation by a sweep of a point Collective Gauss-Seidel relaxation. The latter will smooth the problematic error components.

It might also be useful to perform the point collective relaxation, instead of Distributive, on and near the boundary, thus avoiding the difficulty of imposing boundary conditions for vorticity. 
5. Discussion and conclusions. When discretizing a system of partial differential equations, being concerned just with obtaining an approximation of a certain order of accuracy may not necessarily lead to a satisfactory result. It may be very useful to make sure that the discretization also imitates some fundamental properties of the PDEs. We have outlined a construction of a discretization scheme that not only approximates the compressible Euler equations, but imitates their factorizability property. This paves the way towards construction of optimally efficient multigrid solvers and also should alleviate the problem associated with computation of low-speed flow using the standard shock-capturing schemes.

This paper addressed the subsonic case only. The constructed UHF scheme, though, is related to the family of the genuinely multidimensional upwind schemes constructed previously (see $[18,17,19]$ ). Schemes of this type were demonstrated to have excellent shock capturing capabilities in transonic-supersonic cases together with maintaining the $h$-ellipticity property. Therefore, we do not anticipate any difficulties in extending the factorizable scheme to the transonic-supersonic regimes. This is a subject of future work.

We also developed a relaxation procedure that uses the factorizability property of the scheme for the purpose of obtaining ideal multigrid efficiency. This procedure relies on auxiliary potential and vorticity (or streamfunction) variables. Note that using only an auxiliary potential variable (as in the incompressible case) is not sufficient yet to decouple the different factors (or obtaining upper- or lower-triangular matrix of difference operators). This is due to the bulk viscosity-like terms, which are a part of the artificial dissipation in the constructed scheme and are essential for its factorizability and second order accuracy. Another auxiliary variable is needed - vorticity (or streamfunction).

The approach we introduce in this paper seems very general. The future work will be devoted generalizing it to three dimensions, to Navier-Stokes equations and to unsteady problems.

The proposed approach also essentially unifies the numerical treatment of the steady incompressible and compressible flow, since, on one hand, it belongs to the class of projection-type methods, on the other hand its extension to the supersonic case has shock-capturing capabilities.

Acknowledgments. The author would like to thank Tom Roberts, Jim Thomas, Dimitri Mavriplis and Manny Salas for reading the manuscript and making numerous helpful suggestions and comments.

\section{REFERENCES}

[1] A. A. Amsden and F. H. Harlow, A simplified MAC technique for incompressible fluid flow calculations, J. Comp. Phys., 6 (1970), pp. 322-325.

[2] B. J. BRAAMS, Modelling of a transport problem in plasma physics, in Topics in Applied Numerical Analysis, J. G. Verwer, ed., Centrum voor Wiskunde en Informatica, 1984.

[3] A. BRANDT, Multigrid techniques: 1984 guide with applications to fluid dynamics, The Weizmann Institute of Science, Rehovot, Israel, 1984.

[4] A. Brandt and I. Yavneh, Accelerated multigrid convergence and high Reynolds recirculating flows, SIAM J. Sci. Statist. Comput., 14 (1993), pp. 607-626.

[5] A. J. Chorin, A numerical method for solving incompressible viscous problems, J. Comp. Phys., 2 (1967), pp. 12- 26.

[6] P. COLELLA, Multidimensional upwind methods for hyperbolic conservation laws, Tech. Rep. LBL-17023, Lawrence Berkeley Report, 1984.

[7] _. Multidimensional upwind methods for hyperbolic conservation laws, J. Comp. Phys., 87 (1990), p. 171 . 
[8] F. H. HARLOW AND J. E. WELCH, Numerical calculation of time-dependent viscous incompressible flow of fluid with free surface, Phys. Fluids, 8 (1965), pp. 2182-2189.

[9] R. J. LEVEQue, High resolution finite volume methods on arbitrary grids via wave propagation, J. Comp. Phys., 78 (1988).

[10] S. A. OrszaG, M. Israeli, AND M. O. Deville, Numerical simulations of viscous incompressible flows, Ann. Reviews in Fluid Mech., 6 (1974), pp. 281-318.

[11] S. V. Patankar, Numerical heat transfer and fluid flow, Hemisphere, 1980.

[12] S. V. Patankar and D. B. Spalding, A calculation procedure for heat, mass and momentum transfer in three-dimensional parabolic flow, Int. J. Heat and Mass Transfer, 15 (1972), pp. 1787-1806.

[13] Y. B. RADVoGIN, Quasi-monotonous multidimensional difference schemes with second order accuracy. Soviet Academy of Science, preprint, 1991.

[14] T. W. Roberts, R. C. Swanson, And D. Sidilkover, An algorithm for ideal multigrid convergence for the the steady Euler equations. to appear in Computers \& Fluids, 1997.

[15] D. SidilKover, Some approaches towards constructing optimally efficient multigrid solvers for the inviscid flow equations. ICASE Report No. 97-39, also to appear in Computers \& Fluids.

[16] — Numerical solution to steady-state problems with discontinuities, $\mathrm{PhD}$ thesis, The Weizmann Institute of Science, Rehovot, Israel, 1989.

[17] _ - A genuinely multidimensional upwind scheme and efficient multigrid solver for the compressible Euler equations, ICASE Report No. 94-84, Institute for Computer Applications in Science and Engineering, 1994.

[18] _ A genuinely multidimensional upwind scheme for the compressible Euler equations, in Proceedings of the Fifth International Conference on Hyperbolic Problems: Theory, Numerics, Applications, J. Glimm, M. J. Graham, J. W. Grove, and B. J. Plohr, eds., World Scientific, June 1994.

[19] _ Multidimensional upwinding and multigrid. AIAA 95-1759, June 19-22, 1995. 12th AIAA CFD meeting, San Diego.

[20] D. Sidilkover AND U. Ascher, A multigrid solver for the steady-state Navier-Stokes equations using the pressure-Poisson formulation, Matematica Aplicada e Computational, 14 (1995), pp. 21-35.

[21] D. Sidilkover AND A. BrandT, Multigrid solution to steady-state 2d conservation laws, SIAM J. Numer. Anal., 30 (1993), pp. 249-274.

[22] S. TA'ASAN, Canonical forms of multidimensional steady inviscid flows, ICASE Report No. 93-94, Institute for Computer Applications in Science and Engineering, 1993.

[23] _- Canonical-variables multigrid method for steady-state euler equations, ICASE Report No. 94-14, Institute for Computer Applications in Science and Engineering, 1994. 
Public reporting burden for this colfection of information is estimated to average 1 hour per response, including the time for reviewing instructions, searching existing data sources, gathering and maintaining the data needed, and completing and reviewing the collection of information. Send comments regarding this burden estimate or any other aspect of this collection of information, including suggestions for reducing this burden, to Washington Headquarters Services, Directorate for information Operations and Reports, 1215 Jefferson Davis Highway. Suite 1204. Arlington, VA 22202-4302, and to the Office of Management and Budget, Paperwork Reduction Project (0704-0188). Washington, DC 20503.

\begin{tabular}{|l|l|l}
\hline 1. AGENCY USE ONLY(Leave blank) & $\begin{array}{c}\text { 2. REPORT DATE } \\
\text { June } 1999\end{array}$ & $\begin{array}{c}\text { 3. REPORT TYPE AND DATES COVERED } \\
\text { Contractor Report }\end{array}$ \\
\hline
\end{tabular}

4. TITLE AND SUBTITLE

Factorizable Schemes for the Equations of Fluid Flow

5. FUNDING NUMBERS

C NAS1-97046

WU 505-90-52-01

\section{AUTHOR(S)}

David Sidilkover

7. PERforming organization NAME(S) AND ADDRESS(ES)

Institute for Computer Applications in Science and Engineering

Mail Stop 132C, NASA Langley Research Center

Hampton, VA 23681-2199

8. PERFORMING ORGANIZATION REPORT NUMBER

ICASE Report No. 99-20

9. SPONSORING/MONITORING AGENCY NAME(S) AND ADDRESS(ES)

National Aeronautics and Space Administration

Langley Research Center

Hampton, VA 23681-2199

10. SPONSORING/MONITORING AGENCY REPORT NUMBER

NASA/CR-1999-209345

ICASE Report No. 99-20

\section{SUPPLEMENTARY NOTES}

Langley Technical Monitor: Dennis M. Bushnell

Final Report

Submitted to Applied Numerical Mathematics.

12a. DISTRIBUTION/AVAILABILITY STATEMENT

12b. DISTRIBUTION CODE

Unclassified--Unlimited

Subject Category 64

Distribution: Nonstandard

Availability: NASA-CASI (301) 621-0390

13. ABSTRACT (Maximum 200 wonds)

We present an upwind high-resolution factorizable (UHF) discrete scheme for the compressible Euler equations that allows to distinguish between full-potential and advection factors at the discrete level. The scheme approximates equations in their general conservative form and is related to the family of genuinely multidimensional upwind schemes developed previously and demonstrated to have good shock-capturing capabilities. A unique property of this scheme is that in addition to the aforementioned features it is also factorizable, i.e., it allows to distinguish between full-potential and advection factors at the discrete level. The latter property facilitates the construction of optimally efficient multigrid solvers. This is done through a relaxation procedure that utilizes the factorizability property.

\begin{tabular}{|c|c|c|c|}
\hline \multirow{2}{*}{\multicolumn{3}{|c|}{$\begin{array}{l}\text { 14. SUBJECT TERMS } \\
\text { incompressible and compressible flow, factorizable schemes, genuinely multidimensional } \\
\text { upwind schemes, optimal multigrid efficiency }\end{array}$}} & $\begin{array}{c}\text { 15. NUMBER OF PAGES } \\
18 \\
\end{array}$ \\
\hline & & & $\begin{array}{r}\text { 16. PRICE CODE } \\
\mathrm{A} 03 \\
\end{array}$ \\
\hline $\begin{array}{l}\text { 17. SECURITY CLASSIFICATION } \\
\text { OF REPORT } \\
\text { Unclassified }\end{array}$ & $\begin{array}{l}\text { 18. SECURITY CLASSIFICATION } \\
\text { OF THIS PAGE } \\
\text { Unclassified }\end{array}$ & $\begin{array}{l}\text { 19. SECURITY CLASSIFICATION } \\
\text { OF ABSTRACT }\end{array}$ & $\begin{array}{l}\text { 20. LIMITATION } \\
\text { OF ABSTRACT }\end{array}$ \\
\hline NSN 7540-01-280-5500 & & & $\begin{array}{l}\text { Standard Form 298(Rev. } 2 \\
\text { Prescribed by ANSI Std. Z39-18 } \\
298-102\end{array}$ \\
\hline
\end{tabular}

